\title{
Inhibition of Notch1 promotes hedgehog signalling in a HES1-dependent manner in chondrocytes and exacerbates experimental osteoarthritis
}

\author{
Neng-Yu Lin, ${ }^{1}$ Alfiya Distler, ${ }^{1}$ Christian Beyer, ${ }^{1}$ Ariella Philipi-Schöbinger, ${ }^{1}$ \\ Silvia Breda, ${ }^{1,2}$ Clara Dees, ${ }^{1}$ Michael Stock, ${ }^{1}$ Michal Tomcik, ${ }^{3}$ Andreas Niemeier, ${ }^{4}$ \\ Francesco Dell'Accio, ${ }^{5}$ Kolja Gelse, ${ }^{6}$ Mark P Mattson, ${ }^{7}$ Georg Schett, ${ }^{1}$ \\ Jörg HW Distler ${ }^{1}$
}

\begin{abstract}
Handling editor Tore K Kvien
- Additional material is published online only. To view please visit the journal online (http://dx.doi.org/10.1136/ annrheumdis-2015-208420)

For numbered affiliations see end of article.
\end{abstract}

\section{Correspondence to} Dr Jörg H W Distler, Department of Internal Medicine 3 and Institute for Clinical Immunology, University of Erlangen-Nuremberg, Erlangen D-91054, Germany; joerg.distler@uk-erlangen.de

Received 13 August 2015 Revised 10 December 2015 Accepted 16 January 2016 Published Online First 5 February 2016

\section{CrossMark}

To cite: Lin N-Y, Distler A, Beyer C, et al. Ann Rheum Dis 2016;75:2037-2044.

\section{ABSTRACT}

Objectives Notch ligands and receptors have recently been shown to be differentially expressed in osteoarthritis (OA). We aim to further elucidate the functional role of Notch signalling in OA using Notch1 antisense transgenic (Notch1 AS) mice.

Methods Notch and hedgehog signalling were analysed by real-time PCR and immunohistochemistry. Notch-1 AS mice were employed as a model of impaired Notch signalling in vivo. Experimental OA was induced by destabilisation of the medial meniscus (DMM). The extent of cartilage destruction and osteophyte formation was analysed by safranin- 0 staining with subsequent assessment of the Osteoarthritis Research Society International (OARSI) and Mankin scores and $\mu \mathrm{CT}$ scanning. Collagen $X$ staining was used as a marker of chondrocyte hypertrophy. The role of hairy/enhancer of split 1 (Hes-1) was investigated with knockdown and overexpression experiments.

Results Notch signalling was activated in human and murine OA with increased expression of Jagged1, Notch1, accumulation of the Notch intracellular domain 1 and increased transcription of Hes-1. Notch1 AS mice showed exacerbated OA with increases in OARSI scores, osteophyte formation, increased subchondral bone plate density, collagen $X$ and osteocalcin expression and elevated levels of Epas1 and ADAM-TS5 mRNA. Inhibition of the Notch pathway induced activation of hedgehog signalling with induction of Gli-1 and Gli-2 and increased transcription of hedgehog target genes. The regulatory effects of Notch signalling on Gliexpression were mimicked by Hes-1.

Conclusions Inhibition of Notch signalling activates hedgehog signalling, enhances chondrocyte hypertrophy and exacerbates experimental OA including osteophyte formation. These data suggest that the activation of the Notch pathway may limit aberrant hedgehog signalling in $\mathrm{OA}$.

The Notch pathway is one of the so-called morphogen pathways, which are evolutionary conserved key-regulators of embryonic development. Notch signalling is also crucial for tissue homeostasis in adults. ${ }^{1}$ In mice and humans, four Notch receptors (Notch1-4) and five different ligands have been identified. Binding of these ligands to Notch receptors triggers proteolytic cleavage of
Notch receptors by $\gamma$-secretase to release the Notch intracellular domain (NICD). ${ }^{2}$ The NICD translocates into the nucleus to enhance the transcription of target genes such as hairy/enhancer of split (Hes). ${ }^{3}$ Alterations in Notch signalling have been implicated in the pathogenesis of various diseases such as cancer, neurodegeneration, fibrosis and most recently osteoarthritis (OA) ${ }^{4-6}$ Although general inhibition of Notch signalling was shown to cause serious side effects, ${ }^{7}$ recent studies demonstrate that selective targeting of individual Notch ligands and receptors may open new perspectives and may overcome major toxicity-related limitations of general inhibition of Notch signalling. ${ }^{8} 9$

$\mathrm{OA}$ is characterised by degeneration of articular cartilage, limited intra-articular inflammation with synovitis, changes in periarticular and subchondral bone and osteophyte formation by endochondral ossification. $^{10}{ }^{11}$ Several studies have identified Notch- and Hedgehog-signalling as essential pathways involved in cartilage development and bone remodelling. ${ }^{12-14}$ Notch/Hes1 signalling components were shown to be overexpressed in damaged areas of OA cartilage. ${ }^{15-19}$ Recent in vitro studies reported that genetic inhibition of Notch signalling by deletion of the Notch transcriptional effector RBPj or pharmacologic inhibition of Notch signalling prevented de-differentiation of cultured murine chondrocytes. ${ }^{6} 20$ On the other hand, selective knockout of RBPjк in chondroprogenitor cells impaired endochondral ossification and caused accumulation of hypertrophic chondrocytes with an increased hypertrophic zone relative to the limb length. ${ }^{6}$

Besides the Notch pathway, other morphogen pathways have been implicated in the pathogenesis of OA. Hedgehog signalling is activated in OA chondrocytes and its overexpression promotes chondrocyte hypertrophy and cartilage damage in mice resembling human OA. ${ }^{14}{ }^{21}$ In contrast, pharmacological or genetic inactivation of hedgehog signalling reduces the severity of OA and thus might represent a future treatment option. ${ }^{22} 23$

Individual morphogen pathways are highly crosslinked at multiple levels and regulate each other during embryogenesis and tumourigenesis, and also during tissue homeostasis. ${ }^{24} 25$ Depending on the cellular and molecular context, the cellular effects 
of those pathways as well as the outcome of these interactions can differ and act synergistically as well as antagonistically in different cell-types and tissues.

The purpose of the present study is to elucidate the role of Notch signalling in OA and to characterise the molecular pathways regulated by Notch in murine chondrocytes.

\section{MATERIALS AND METHODS}

\section{Human knee joint cartilage}

Cartilage was obtained from 5 patients with trauma and 12 patients with OA undergoing knee replacement surgery (four males and eight females, mean age: 70.2 \pm 7.3 years (range: 58-80)). Detailed patient information and Osteoarthritis Research Society International (OARSI) scores are listed in online supplementary table S1. Representative safranin-O stainings are shown in online supplementary figure S1. Tissue samples were obtained from osteoarthritic areas and compared with samples of macroscopically and microscopically normal areas from the same patient.

\section{Animals and treatments}

Male Notch-1 antisense transgenic (Notch1 AS) mice on a C57BL/6 mice background were analysed. Notch1 AS mice have been described. ${ }^{4} 26$ These mice appear phenotypically normal, but protein levels of Notch-1 are reduced by $50-60 \%$.

\section{Destabilisation of the medial meniscus model}

Experimental OA was induced by surgical destabilisation of the medial meniscus (DMM) in 8-week-old male wildtype mice or Notch1 AS mice by DMM as described previously. ${ }^{27}{ }^{28}$ Mice were anaesthetised with $25 \%$ Xylazine $(20 \mathrm{mg} / \mathrm{mL})$ and $25 \%$ Ketavet $(100 \mathrm{mg} / \mathrm{mL})$ in $60 \mu \mathrm{L}$ phosphate-buffered saline (PBS) by intraperitoneal injection. Hind limbs were shaved and prepared for aseptic surgery. The knee joint was exposed through a medial parapatellar approach and lateral dislocation of the patella. The medial meniscotibial ligament was carefully transected. After 8 weeks, mice were sacrificed and analysed ( $n=8$ per group).

\section{Histomorphometric analysis}

The knee joints were fixed in 4\% paraformaldehyde for $24 \mathrm{~h}$. Decalcified and paraffin-embedded knee joints were cut in $5 \mu \mathrm{m}$ frontal sections through the entire joint at $70 \mu \mathrm{m}$ intervals. Sections were stained with $0.1 \%$ safranin-O solution and 0.001\% Fast Green solution (Sigma-Aldrich, St. Louis, Missouri, USA). Each knee yielded 12 slides for scoring by blinded observers using modified Mankin ${ }^{29}$ and OARSI scores. ${ }^{30} 31$ The average histological grading scores in the medial femoral condyle (MFC) and the medial tibial plateau (MTP) were analysed in five safranin-O stained sections at intervals of $70 \mu \mathrm{m}$ in each mouse knee. To quantify osteoclast number in subchondral bone, haematoxylin and tartrate-resistant acid phosphatase (TRAP) staining was performed, using a leucocyte acid phosphatase staining kit (Sigma-Aldrich). ${ }^{32}$

\section{Osteophyte grading score}

For evaluation of osteophyte size, the thickness of the osteophyte was compared with that of the adjacent articular cartilage and classified in four categories $(0$, none; 1 , small, the same thickness as the adjacent articular cartilage; 2, medium, one to three times the thickness of the adjacent articular cartilage; 3, large, more than three times the thickness of the adjacent articular cartilage). Osteophyte maturity is classified in five categories (0, none; 1 , precartilagious lesion; 2 , predominantly cartilage; 3 , mixed cartilage and bone; 4, predominantly bone) as described. $^{30}{ }^{33}$ The average histological grading scores in MFC

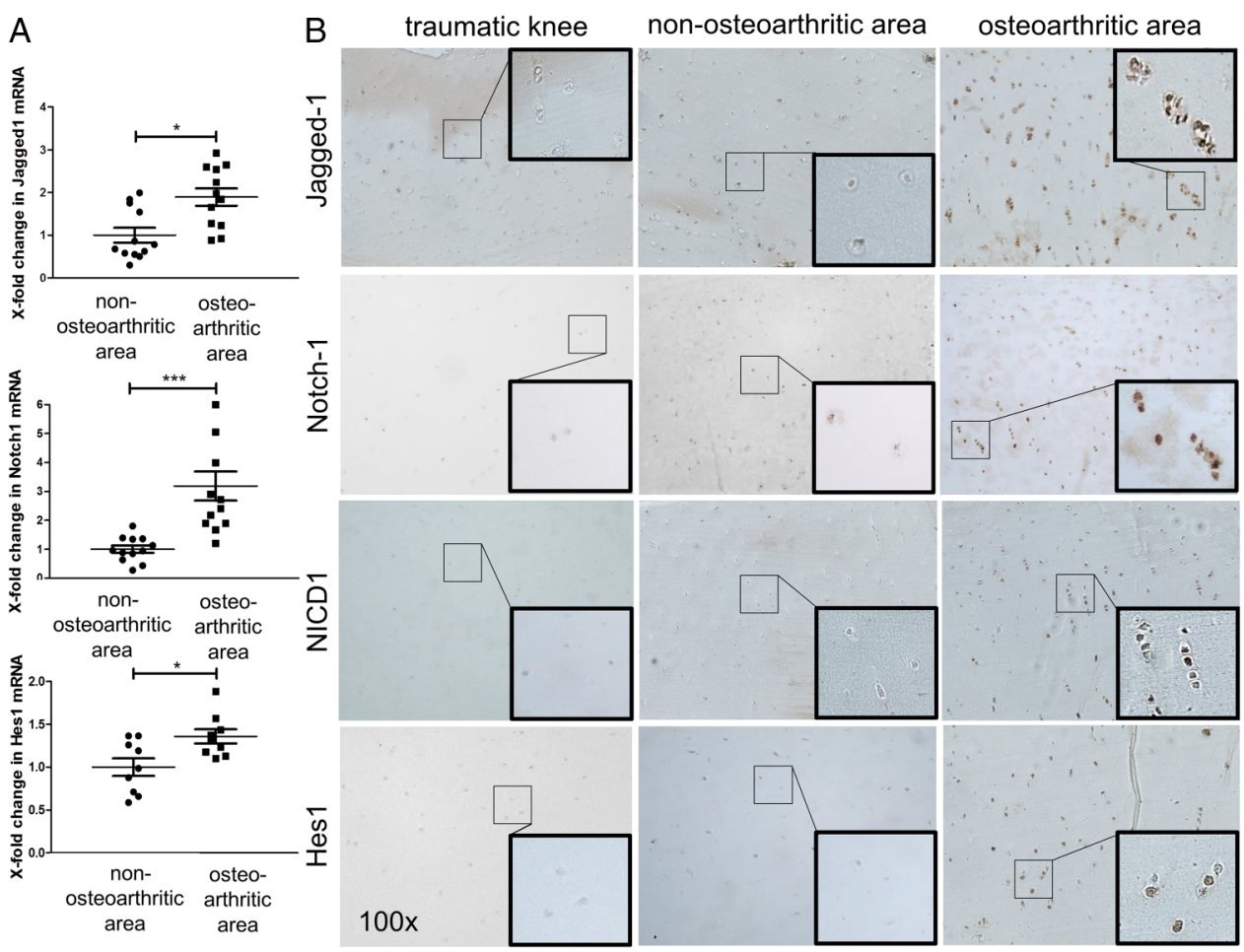

Figure 1 Notch1 signalling pathway is activated in patients with osteoarthritis. (A) Quantification of mRNA levels of Jagged1, Notch1 and Hes-1 in osteoarthritic areas of human knee cartilage compared with macroscopically and microscopically healthy areas from the same patient and patients with trauma as control groups. (B) Representative images of immunohistochemistry for Jagged1, Notch1, Hes1 and Notch intracellular domain (NICD) 1 in human samples are shown at 100 -fold magnification. *Statistically significant differences with $p<0.05$. ${ }^{* *}$ Statistically significant differences with $\mathrm{p}<0.005$. 
and MTP were evaluated in five sections at intervals of $70 \mu \mathrm{m}$ in each mouse knee, and the average scores were summed.

\section{Immunohistochemical staining}

Sections were incubated with polyclonal rabbit anti-human NICD1 antibodies, anti-Notch1 antibodies (Abcam, Cambridge, UK), polyclonal goat anti-Jag1 antibodies (Santa Cruz Biotechnology, Santa Cruz, California, USA), polyclonal rabbit anti-Hes1 antibodies (Merck Millipore), monoclonal mouse anti Gli1 and Gli2 antibodies (Abnova, Heidelberg, Germany), monoclonal mouse anti-collagen $\mathrm{X}$ antibodies (clone X53, a kind gift from Klaus Von-der-Mark) and polyclonal rabbit antiosteocalcin antibodies (Merck Millipore). ${ }^{34}$ Irrelevant isotype antibodies were used as negative controls (Abcam). Biotinylated goat anti-rabbit or goat anti-mouse antibodies (DAKO, Hamburg, Germany) and streptavidin-biotin ABC Kits (Biozol, Eching, Germany) were used as secondary antibodies. The evaluations were performed in a blinded manner.

\section{Immunofluorescence staining}

Knee joint sections from C57BL/6 wildtype sham and DMM operated mice were incubated with polyclonal rabbit anti NICD1 antibodies (Abcam), monoclonal mouse anti Gli1 and Gli2 antibodies (Abnova). Irrelevant isotype antibodies (Abcam) were used as negative controls. Polyclonal goat anti-rabbit antibodies labelled with fluorescent dye Alexa Fluor 488 (Invitrogen, Darmstadt, Germany) were used as secondary antibodies. Quantification was performed with ImageJ software (V.1.41; National Institutes of Health) and the intensity of immunofluorescence was analysed with equal conditions for all samples within the respective experiments. Exposure times as well as light intensity were the same throughout all samples within an experiment.

\section{Cell cultures}

Human T/C28a2 chondrocytic cells were grown $\left(37^{\circ} \mathrm{C}\right.$ in $5 \%$ CO2) in 1:1 Ham's F-12/Dulbecco's Modified Eagle's medium (DMEM) supplemented with 10\% Fetal Bovine Serum (FBS) (Thermo Fisher, Waltham, Massachusetts, USA), $2 \mathrm{mM}$ glutamine, $100 \mathrm{U} / \mathrm{mL}$ penicillin, $100 \mathrm{mg} / \mathrm{mL}$ streptomycin (Lonza, Basel, Switzerland).

\section{siRNA delivery and plasmid constructs}

In overexpression assays, $1 \times 10^{6} \mathrm{~T} / \mathrm{C} 28 \mathrm{a} 2$ cells were transfected with $10 \mu \mathrm{g}$ of human Hes-1 plasmid (a gift from Linzhao Cheng, Addgene plasmid \# 17624) ${ }^{35}$ and $10 \mu \mathrm{g}$ of backbone plasmid using Lipofectamine Plus Reagent (Invitrogen, Germany). In silencing assays, $1 \times 10^{6} \mathrm{~T} / \mathrm{C} 28 \mathrm{a} 2$ cells were transfected with $1 \mu \mathrm{g}$ of human Hes-1 siRNA, Notch1 siRNA or non-targeting sequences (Santa Cruz Biotechnology, Germany). Cell lysates were collected after $48 \mathrm{~h}$. The efficacy of lipofectamine-mediated transfection in overexpression as well as knockdown experiments was analysed by western blot and showed changes in gene expression of a minimum of $70 \%$ for siRNA. Cellular survival was monitored with MTT ([3-(4,5-Dimethylthiazol-2-yl)-2,5-Diphenyltetrazolium Bromide]) assays.

\section{Western blot analysis}

Western blots were performed according to common protocols, ${ }^{36}$ using polyclonal rabbit Notch1 antibodies (Abcam),
A
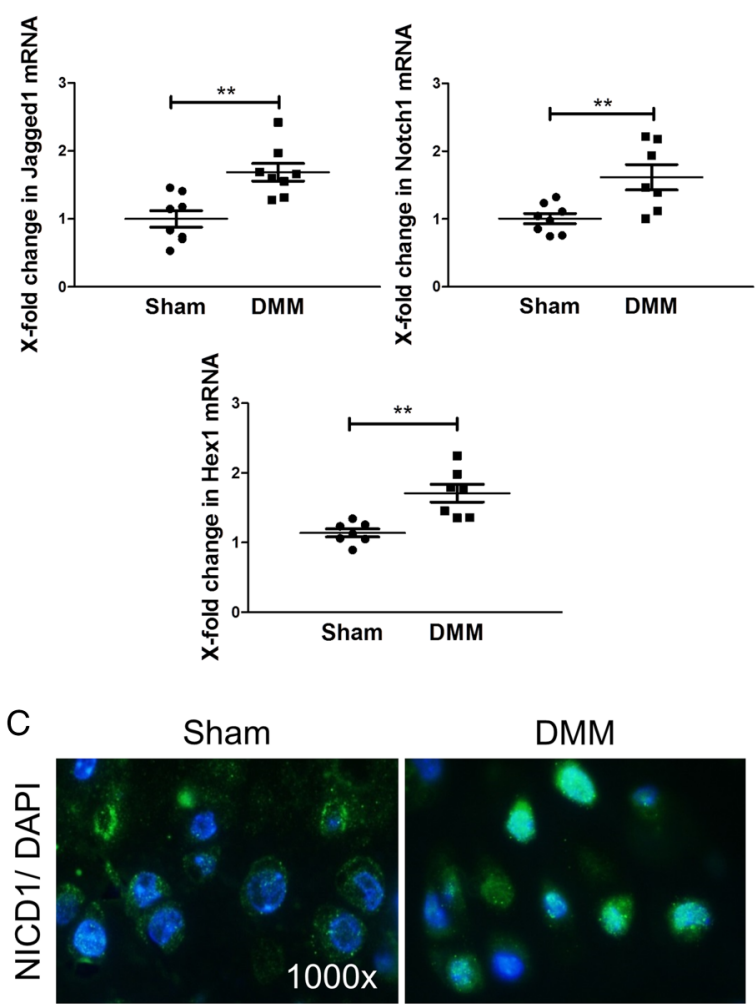

B

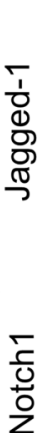

Sham

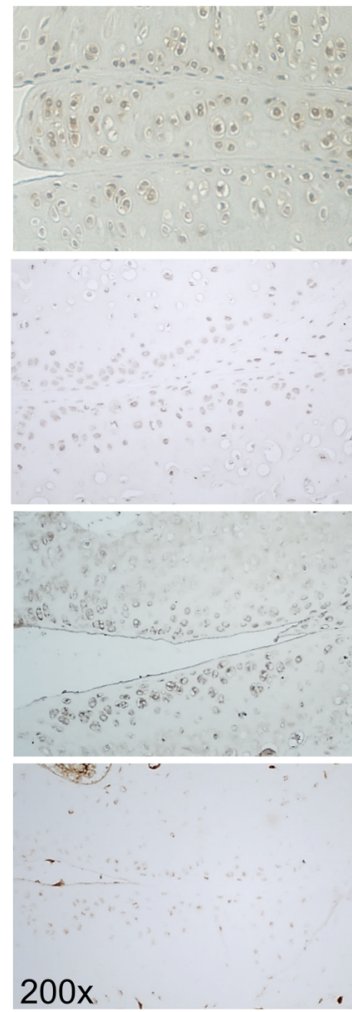

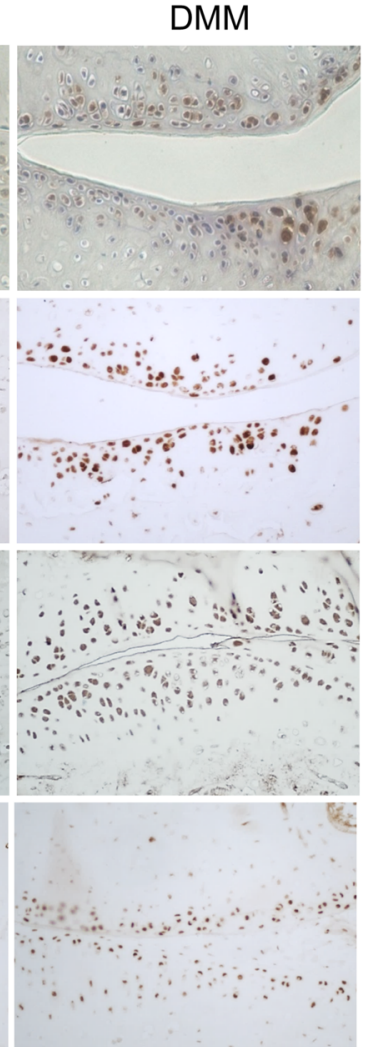

Figure 2 Notch1 signalling is activated in the destabilisation of the medial meniscus (DMM) model of osteoarthritis (OA). (A) Quantification of mRNA levels of Jagged1, Notch1 and Hes-1 in entire knee epiphysis of mice undergoing DMM. (B) Representative images of immunohistochemistry staining for Jagged1, Notch1, Hes1 and Notch intracellular domain (NICD) 1 in the DMM model of murine articular cartilage are shown at 100-fold magnification. (C) Representative images of immunofluorescence staining for the nuclear accumulation of NICD1 in the articular chondrocytes of DMM model of murine $O A$ are shown at 1000 -fold magnification. $n=6$ for each group. ${ }^{*}$ Statistically significant differences with $p<0.01$. 
polyclonal rabbit anti-Hes1 antibodies (Merck Millipore), monoclonal mouse anti Gli1 and Gli2 antibodies (Abnova) and horseradish peroxidase-conjugated polyclonal goat anti-rabbit (DAKO). $\beta$-actin served as loading control.

\section{Quantitative real-time PCR}

Total RNA was isolated from the entire knee epiphysis using a NucleoSpin RNA isolation kit (Machery-Nagel, Düren, Germany). Gene expression was quantified by N',N'-dimethylN-[4-[(E)-(3-methyl-1,3-benzothiazol-2-ylidene)methyl]-1-phenylquinolin-1-ium-2-yl]-N-propylpropane-1,3-diamine (SYBR) Green real-time PCR using the MxPro 3005P qPCR System (Agilent Technologies, Santa Clara, California, USA). $\beta$-actin was used to normalise for the amounts of cDNA within each sample and all primers are listed in the online supplementary table S2 ( $n=3$ independent experiments with three replicates each).

\section{Statistical analysis}

All results were tested for normal distribution using the D'Agostino \& Pearson omnibus normality test. In case of normal distribution, results were further evaluated using the student's $t$ test. For not normally distributed samples, a MannWhitney $U$ test was performed. Data are represented as box blots with median and IQR or as dot blots using GraphPad Prism V.4.0 Software (GraphPad Software, San Diego, California, USA).

\section{RESULTS}

\section{Notch1 signalling is activated in $\mathrm{OA}$ chondrocytes}

We first analysed the mRNA levels of Notch ligands and their receptors in osteoarthritic and relatively healthy areas of human knee cartilage. We observed increased mRNA levels of Jagged-1 and its receptor Notch1 in osteoarthritic areas of human cartilage that were more pronounced in older patients with higher OARSI scores (see figure $1 \mathrm{~A}$ and online supplementary figure S2), but there were no significant changes in Notch2 and Notch4 receptors (see online supplementary figure S3). Notch3 mRNA levels were also slightly increased (see online supplementary figure S3), but the expression levels of Notch3 were much lower than those of Notch1. Moreover, the mRNA levels of the Notch target gene Hes-1 were also significantly increased in osteoarthritic areas (figure 1A). The activation of Notch signalling in human OA was further confirmed by demonstrating increased expression of Jagged-1, Hes1 protein and accumulation of the NICD1 as the active signalling molecule in OA chondrocytes of the intermediate zone of knee cartilage by immunohistochemistry (figure 1B). Notch signalling was also activated in murine $\mathrm{OA}$ induced by DMM with increased mRNA levels of Jagged-1, Notch1 and Hes-1 (figure 2A), overexpression of Jagged-1, Notch1, Hes1 protein (figure 2B) and nuclear accumulation of the NICD1 (figure 2C).

\section{Increased severity of OA in Notch1 AS mice}

To evaluate the functional role of Notch signalling in OA in vivo, experimental OA was induced by DMM in Notch1 AS mice and wildtype littermates. The expression of Notch1 was reduced by $65 \%$ in chondrocytes of Notch 1 AS mice compared with control mice (see online supplementary Figures S4 and S5). No histological differences in cartilage or joint architecture were observed in sham operated Notch1 AS mice (figure 3A). However, Notch1 AS mice developed more severe OA upon surgical DMM with significantly higher modified Mankin and
A

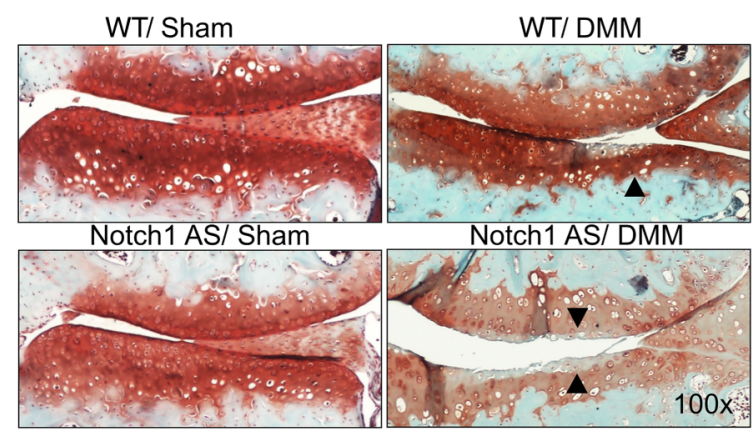

C

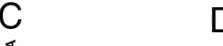

Epas1

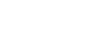

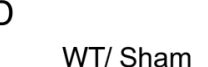
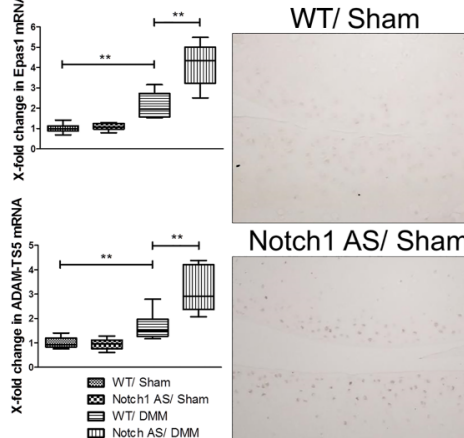

WT/ DMM

B

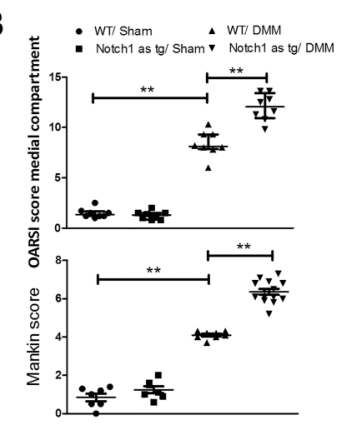

ADAMTS-5

Figure 3 Notch1 antisense transgenic (Notch1 AS) mice develop more severe osteoarthritis (OA). (A) Representative images of medial compartment of knee sections from wildtype (WT) littermates and Notch1 AS mice undergoing surgical destabilisation of the medial meniscus (DMM) or sham operations stained with safranin-0 and fast green (100-fold magnification). (B) Osteoarthritic changes in knee joints as quantified with the modified Mankin and Osteoarthritis Research Society International (OARSI) score. (C) Quantification of mRNA levels of Epas1 and ADAM-TS5 in entire knee epiphysis of WT and Notch1 AS mice with DMM. (D) Quantification of protein levels of Epas1 and ADAM-TS5 in articular cartilage of WT and Notch1 AS mice with DMM. $n=8$ per group. * * Statistically significant differences with $p<0.01$. 
OARSI scores compared with wildtype littermates (figure 3A, B). The mRNA and protein levels of Epas1 and ADAM-TS5, two key-players in the pathogenesis of OA, were significantly higher in the cartilage of Notch1 AS mice undergoing DMM compared with controls (figure 3D). Moreover, increased size and maturity of osteophytes were observed in the cartilage of Notch1 AS mice upon DMM (figure 4A). $\mu$ CT showed increased density of the subchondral bone plate (SBP density) in Notch1 AS mice without differences in subchondral bone thickness (figure 4B). To further assess osteophyte formation and remodelling of the SBP, the numbers of osteoclasts and osteoblasts at both sites were assessed. Consistent with enhanced bone remodelling, the number of TRAP-positive osteoclasts as well as the number of osteocalcin-positive osteoblasts was increased in Notch1 AS mice undergoing DMM compared with control mice (figure 4C, D). We also observed increased staining for type $\mathrm{X}$ collagens as a marker of hypertrophic chondrocytes (figure 4C).

\section{Inhibition of Notch signalling enhances hedgehog signalling in experimental $O A$}

Notch and hedgehog pathways regulate each other during development and hedgehog signalling has been identified as a key pathway in OA. ${ }^{14}$ We therefore hypothesised that the exacerbation of OA in Notch1 AS mice may result from activated hedgehog signalling. Indeed, we found that the mRNA levels of Gli-1 and Gli-2, two downstream transcription factors of hedgehog signalling, were increased in Notch1 AS mice with DMM compared with wildtype mice with DMM (figure 5A). We also observed increased staining for Gli-1 and Gli-2 in chondrocytes of Notch1 AS mice (figure 5C). Moreover, the mRNA levels of the hedgehog target genes Ptch1 and Ptch2 were also elevated in Notch1 AS mice (figure 5A). After demonstrating an upregulation of hedgehog signalling with accumulation of Gli transcription factors in chondrocytes, we further analysed the underlying molecular mechanisms. We confirmed the upregulation of Gli-1 and Gli-2 by siRNA-mediated knockdown of Notch-1-cultured chondrocytes (figure 5B). In particular, we aimed to characterise the role of the basic helix-loop-helix (bHLH) transcription factor Hes1 as a downstream mediator of Notch-signalling. siRNA-mediated knockdown of Hes-1 alone was sufficient to induce Gli-1 and, to a less extent, Gli-2 expression in cultured chondrocytes (figure 5B). In contrast, overexpression of Hes-1 reduced the levels of Gli-1 and Gli-2, providing evidence that the regulatory effects of Notch on Hedgehog signalling are dependent on Hes-1 (figure 5B).

\section{DISCUSSION}

Notch signalling has been proposed as a potential target for the treatment of $\mathrm{OA}^{20}$ based on the findings that (i) inhibition of Notch signalling in cultured murine chondrocytes by
A

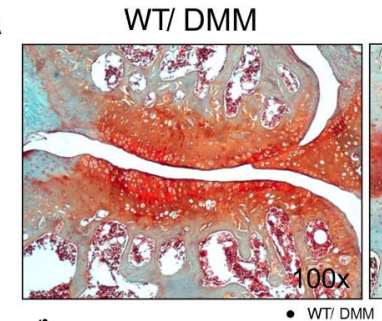

Notch1 AS/ DMM

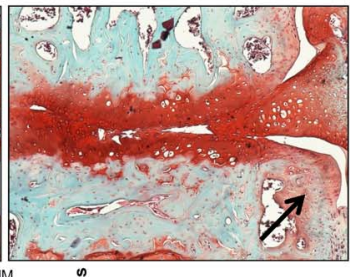

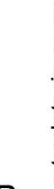
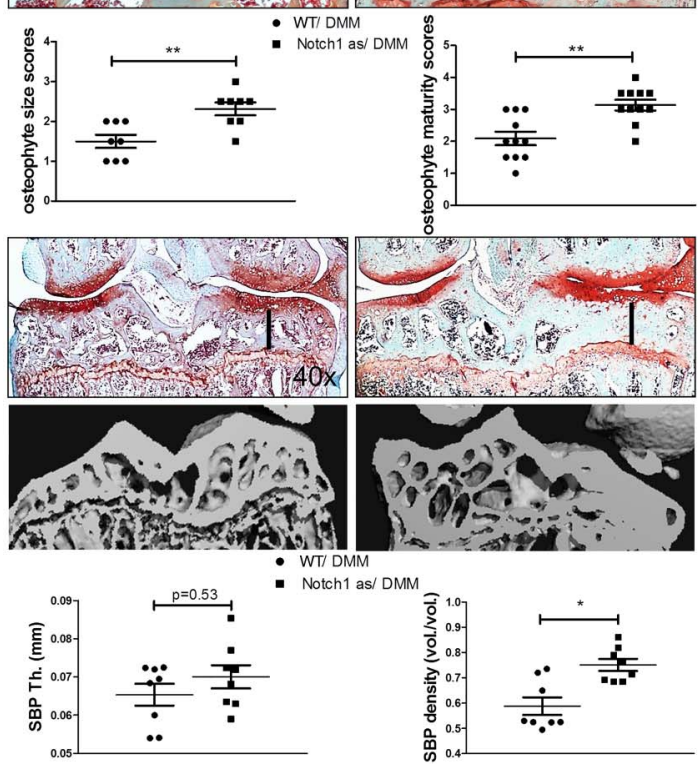

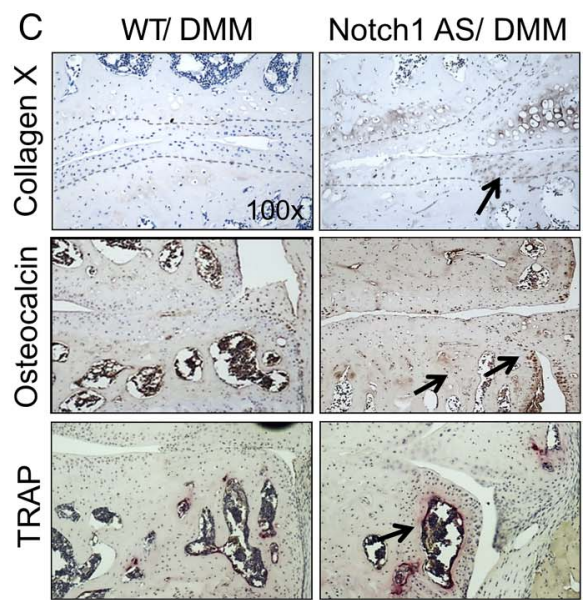

D

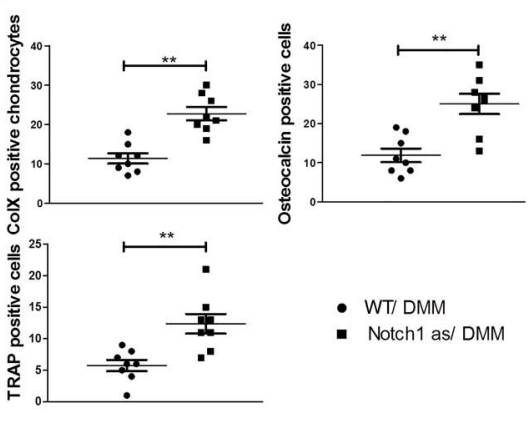

Figure 4 Osteophyte formation upon destabilisation of the medial meniscus (DMM) is exacerbated in Notch1 antisense transgenic (Notch1 AS) mice. (A) Representative images of knee sections from wildtype (WT) littermates and Notch1 AS mice undergoing DMM stained with safranin-0. Osteophyte formation is indicated by arrows (100-fold magnification). The quantification of osteophyte size and maturity is shown in the lower panel. (B) Representative images of $\mu \mathrm{CT}$ scans and safranin- 0 stainings showing the thickness and the density of subchondral bone plate (indicated by vertical black bars, 40- and 100-fold magnification); the quantification is shown in the lower panel. (C) Representative images of immunohistochemistry staining for Col X and osteocalcin and tartrate-resistant acid phosphatase (TRAP) staining from WT littermates and Notch1 AS mice with hypertrophic chondrocytes, increased osteocalcin expression and enhanced osteoclastogenesis (indicated by arrow, 100-fold magnification). (D) The quantification of positive stained chondrocytes is shown in the lower panel. $n=8$ per group, *Statistically significant differences with a $\mathrm{p}<0.05$. * ${ }^{*}$ Statistically significant differences with a $\mathrm{p}<0.01$. 
A

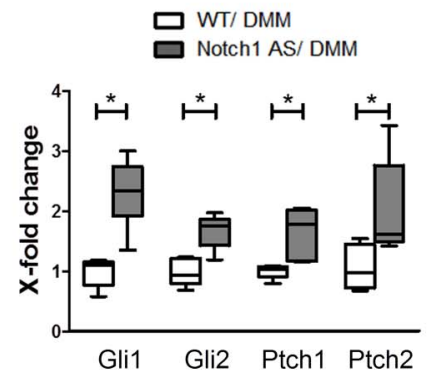

C
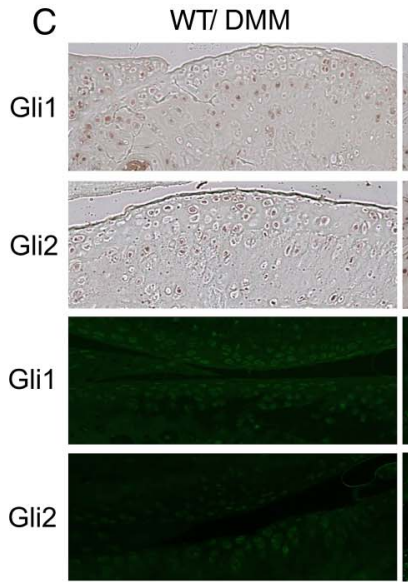

B

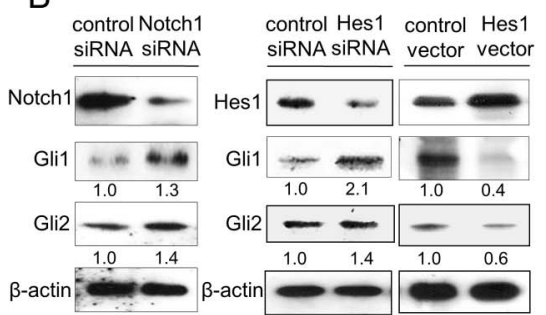

D

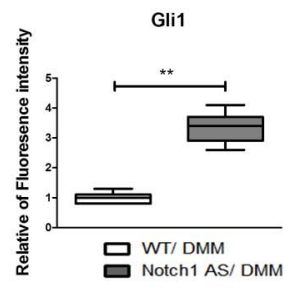

Notch1 AS / DMM

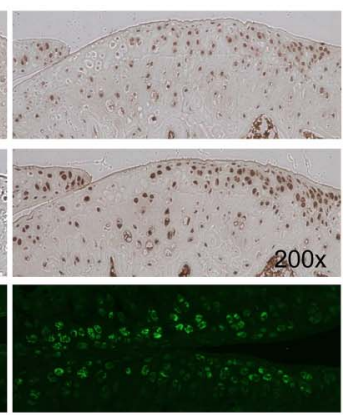

$400 x$

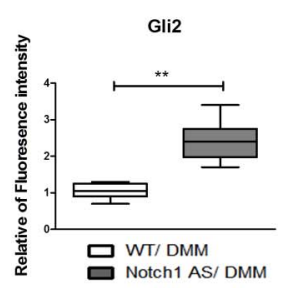

Figure 5 Levels of Notch signalling and Hes-1 are inversely correlated with hedgehog signalling and the expression of Gli-1 and Gli-2. (A) Quantification of mRNA levels of Gli-1, Gli-2, Ptch-1 and Ptch-2 in entire knee epiphysis of wildtype (WT) and Notch1 antisense transgenic (Notch1 AS) mice undergoing destabilisation of the medial meniscus (DMM). (B) Protein levels of Gli-1 and Gli-2 in human T/C28a2 chondrocytic cells transfected with siRNA for Notch1, Hes-1 or Hes-1 expression plasmids. (C) Representative immunohistochemistry and immunofluorescence stainings for Gli-1 and Gli-2 in knee sections from Notch1 AS mice and WT littermates undergoing DMM are shown at 200- and 400-fold magnification. (D) Quantification of relative fluorescence intensity. $n=8$ per group. * Statistically significant differences with a $p<0.05$. ${ }^{*}$ Statistically significant differences with a $\mathrm{p}<0.01$.

$\mathrm{N}$-[(3,5-Difluorophenyl)acetyl]-L-alanyl-2-phenyl]glycine-1,1dimethylethyl ester (DAPT) delayed terminal dedifferentiation, ${ }^{13} 37$ (ii) Notch signalling is activated in chondrocytes of patients with $\mathrm{OA}^{16}{ }^{17}$ and (iii) small molecule inhibitors have already been tested in patients with cancer and would thus be available for clinical trials. However, the results on targeting Notch signalling in experimental $\mathrm{OA}$ are less uniform. Conditional inactivation of RBPj or Hes-1 in chondroprogenitor cells (using a Col2CreER line) ameliorated experimental OA. ${ }^{6} 18$ On the other hand, conditional inactivation of RBPj in mesenchymal joint progenitors (using a PRX1Cre line) induced progressive cartilage degradation. Moreover, mice with inactivation of RBPj in adult chondrocytes (using a Col2CreER line) developed an age-related OA-like pathology. ${ }^{38}$ In the present study, we selectively target Notch 1 as the major Notch receptor in osteoarthritic chondrocytes instead of targeting RBPj as a downstream effector that can also cross-regulate prosurvival signalling cascades by NF-kB and v-myc avian myelocytomatosis viral oncogene homolog (MYC) via loss of RBPJ-mediated repression. ${ }^{39}$ Another difference is the ubiquitous inhibition of Notch signalling compared with the cell type-specific inactivation of RBPj.

In Notch1 AS mice, the selective inhibition of Notch1 exacerbated experimental OA. The modified Mankin and OARSI scores, the number of hypertrophic chondrocytes expressing Collagen X, and the levels of key players in the pathogenesis of $\mathrm{OA}$ such as Epas1 and Aggrecanase1 were all significantly increased in Notch1 AS mice upon DMM. Although a limitation of our study is that we used rather young mice that may not optimally mimic OA in older patients, these studies together demonstrate that Notch-signalling outcomes in the osteoarthritic joint are highly cell type specific with partially opposite effects in mesenchymal precursor cells, chondroprogenitor cells and mature adult chondrocytes. Moreover, the outcome of Notch signalling has most recently been shown to depend on the duration of the activation. While sustained Notch signalling in joint cartilage led to a progressive osteoarthritic-like pathology, transient Notch activation promoted joint maintenance. ${ }^{40}$ Our results and those of Liu et a ${ }^{40}$ suggest that inhibition of Notch signalling may exacerbate, rather than ameliorate $\mathrm{OA}$ in certain conditions. These findings warrant further investigations on the effect of persistent and temporary activation/inactivation of Notch signalling in different cell populations, during different stages of OA before targeting Notch signalling in humans in a non-cell type-specific manner, for example, by treatment with small molecule inhibitors.

We demonstrated that inhibition of Notch signalling promotes osteophyte formation upon DMM. Enhanced osteophyte formation in Notch1 AS mice was accompanied by increased osteoblast activity. Indeed, Notch signalling has been shown to negatively regulate osteoblast and osteoclast activities and promote bone formation in mouse models of rheumatoid arthritis. Inhibition of Notch signalling promotes osteoblast activity, while activation of Notch signalling inhibits osteoblast function in vitro. ${ }^{41}$ Moreover, targeted inactivation of Hes1 in osteoblasts increased the trabecular bone volume and the trabecular number, ${ }^{42}$ while inhibition of Notch signalling ameliorated tumour necrosis factor alpha-induced bone loss and stimulated osteoblast activity in mice. ${ }^{43}$ As described previously, subchondral bone formation was also accompanied by locally enhanced osteoclastogenesis. ${ }^{44}$ Our findings of increased osteoclastogenesis in Notch1 AS mice are consistent with previous studies demonstrating inhibitory effects of Notch signalling on osteoclastogenesis. Deletion of Notch1 in macrophages enhanced osteoclast differentiation, whereas incubation with 
Notch ligands suppressed osteoclastogenesis by downregulation of the receptor for macrophage colony-stimulating factor (M-CSF). ${ }^{45} 46$

The exacerbation of experimental OA in Notch1 AS mice may be caused by upregulation of hedgehog signalling. Hedgehog signalling has been identified as a core pathway in $\mathrm{OA}$, and activation of hedgehog signalling induces an OA-like pathology, while inhibition of hedgehog signalling ameliorates experimental OA. ${ }^{14} 22{ }^{23}$ Hedgehog and Notch signalling are highly interwoven and regulate each other. The interaction between both pathways is best exemplified in the colonic crypts, where Notch activity is observed in stem cells in the base of the crypts, whereas hedgehog signalling is turned on during differentiation and peaks at the luminal site of the crypt. ${ }^{47}$ We demonstrate that inhibition of Notch signalling further enhances the activation of hedgehog signalling in $\mathrm{OA}$ with increased accumulation of the hedgehog transcription factors Gli1 and Gli2. The prominent activation of hedgehog signalling in chondrocytes upon inhibition of Notch may thus promote chondrocyte hypertrophy and exacerbation of the DMM-induced pathology.

Although interactions between Notch and hedgehog pathways are well known, the molecular mechanisms underlying these interactions are incompletely understood. ${ }^{48}$ We provide evidence that Notch may regulate hedgehog signalling in osteoarthritic chondrocytes via the transcription factor Hes- 1. Overexpression of Hes-1 in chondrocytes downregulated the expression of Gli transcription factors, while knockdown of Hes-1 increased the levels of Gli1 and Gli2. Consistent with these findings, Hes- 1 can bind to promoter of Gli proteins to regulate their expression in cancer cells. ${ }^{49} 50$

In summary, we demonstrate that inhibition of Notch signalling by overexpression of a Notch1 antisense construct promotes activation of hedgehog signalling in osteoarthritic chondrocytes in a Hes-1 dependent manner to exacerbate experimental OA. Considering the opposing effects of Notch signalling during different stages of chondrocyte maturation and dose-dependent effects, further investigations are required before modulation of Notch signalling can be considered and enter the phase of clinical trials.

\section{Author affiliations}

${ }^{1}$ Department of Internal Medicine III and Institute for Clinical Immunology, University of Erlangen-Nuremberg, Erlangen, Germany

${ }^{2}$ Division of Rheumatology, University of Pavia, IRCCS Policlinico San Matteo Foundation, Pavia, Italy

${ }^{3}$ Institute of Rheumatology and Department of Rheumatology, 1st Faculty of Medicine, Charles University, Prague, Czech Republic

${ }^{4}$ Department of Orthopedics, University Medical Center Hamburg-Eppendorf, Hamburg, Germany

${ }^{5}$ William Harvey Research Institute, Barts and The London, School of Medicine and Dentistry, Queen Mary, University of London, London, UK

${ }^{6}$ Division of Trauma Surgery and Orthopedic Surgery, Department of Surgery, University of Erlangen-Nuremberg, Germany

${ }^{7}$ Laboratory of Neurosciences, National Institute on Aging Intramural Research Program, Baltimore, Maryland, USA
}

Acknowledgements We especially thank Dr Thelonius Hawellek and Dr Jan Hubert from University Medical Center Hamburg-Eppendorf for helping to provide healthy cartilage samples. We also thank Verena Wäsch, Regina Kleinlein, Katja Dreißigacker and Stefan Fritz for excellent technical assistance.

Contributors Design of the study: N-YL, JHWD. Acquisition of data: N-YL, $A D, C B$, $A P S, C D, M T, F D, K G$. Interpretation of data: N-YL, AD, APS, CB, CD, MT, FD, KG, OD, GS, JHWD. Manuscript preparation: N-YL, JHWD. Provided samples and mice: AN, KG, MPM.

Funding This study was supported by grants A57, J29 and J39 of the Interdisciplinary Center of Clinical Research (IZKF) in Erlangen, grants from the German Research Foundation (DI 1537/5-1, DI 1537/7-1, DI 1537/8-1, DI 1537/ 9-1, DE 2414/2-1 of the Deutsche Forschungsgemeinschaft), a grant of the
ELAN-Foundation Erlangen to NYL and project grants 00023728 from the Ministry of Health of the Czech Republic and SVV262512. The study was also supported by the IMI-funded project BTCure, the carrier support award of the Ernst-Jung Foundation, the BMBF project ANCYLOSS and the Intramural Research Program of the National Institute on Aging. Neng-Yu Lin had a doctoral scholarship by Bavarian Research Foundation.

Competing interests JHWD has consultancy relationships and/or has received research funding from Actelion, BMS, Celgene, Bayer Pharma, Boehringer Ingelheim, JB Therapeutics, Sanofi-Aventis, Novartis, UCB, GSK, Array Biopharma and Active Biotech in the area of potential treatments of SSC and is stock owner of 4D Science $\mathrm{GmbH}$.

Provenance and peer review Not commissioned; externally peer reviewed.

\section{REFERENCES}

1 Guruharsha KG, Kankel MW, Artavanis-Tsakonas S. The Notch signalling system: recent insights into the complexity of a conserved pathway. Nat Rev Genet 2012;13:654-66.

2 Mumm JS, Schroeter EH, Saxena MT, et al. A ligand-induced extracellular cleavage regulates gamma-secretase-like proteolytic activation of Notch1. Mol Cell 2000;5:197-206.

3 Jarriault $S$, Brou $C$, Logeat $F$, et al. Signalling downstream of activated mammalian Notch. Nature 1995:377:355-8.

4 Dees $C$, Zerr $\mathrm{P}$, Tomcik $\mathrm{M}$, et al. Inhibition of Notch signaling prevents experimental fibrosis and induces regression of established fibrosis. Arthritis Rheum 2011;63:1396-404.

5 Kopan R, Goate A. A common enzyme connects notch signaling and Alzheimer's disease. Genes Dev 2000;14:2799-806.

6 Hosaka Y, Saito T, Sugita S, et al. Notch signaling in chondrocytes modulates endochondral ossification and osteoarthritis development. Proc Natl Acad Sci USA 2013;110:1875-80.

7 Ryeom SW. The cautionary tale of side effects of chronic Notch1 inhibition. I Clin Invest 2011;121:508-9.

8 Tran IT, Sandy AR, Carulli AJ, et al. Blockade of individual Notch ligands and receptors controls graft-versus-host disease. J Clin Invest 2013;123:1590-604.

9 Wu Y, Cain-Hom C, Choy L, et al. Therapeutic antibody targeting of individual Notch receptors. Nature 2010;464:1052-7.

10 Goldring MB, Goldring SR. Osteoarthritis. J Cell Physiol 2007;213:626-34.

11 Saito T, Fukai A, Mabuchi A, et al. Transcriptional regulation of endochondral ossification by HIF-2alpha during skeletal growth and osteoarthritis development. Nat Med 2010;16:678-86.

12 Mead TJ, Yutzey KE. Notch signaling and the developing skeleton. Adv Exp Med Biol 2012:727:114-30.

13 Kohn A, Dong Y, Mirando AJ, et al. Cartilage-specific RBPjk-dependent and -independent Notch signals regulate cartilage and bone development. Development 2012;139:1198-212.

14 Lin $A C$, Seeto BL, Bartoszko JM, et al. Modulating hedgehog signaling can attenuate the severity of osteoarthritis. Nat Med 2009;15:1421-5.

15 Grogan SP, Olee T, Hiraoka K, et al. Repression of chondrogenesis through binding of notch signaling proteins HES-1 and HEY-1 to N-box domains in the COL2A1 enhancer site. Arthritis Rheum 2008:58:2754-63.

16 Karlsson C, Brantsing C, Egell S, et al. Notch1, Jagged1, and HES5 are abundantly expressed in osteoarthritis. Cells Tissues Organs (Print) 2008;188:287-98.

17 Mahjoub M, Sassi N, Driss M, et al. Expression patterns of Notch receptors and their ligands in human osteoarthritic and healthy articular cartilage. Tissue Cell 2012:44:182-94.

18 Sugita S, Hosaka Y, Okada K, et al. Transcription factor Hes1 modulates osteoarthritis development in cooperation with calcium/calmodulin-dependent protein kinase 2. Proc Natl Acad Sci USA 2015;112:3080-5.

19 Wang H, Tian Y, Wang J, et al. Inflammatory cytokines induce NOTCH signaling in nucleus pulposus cells: implications in intervertebral disc degeneration. J Biol Chem 2013:288:16761-74.

20 Sassi N, Laadhar L, Driss M, et al. The role of the Notch pathway in healthy and osteoarthritic articular cartilage: from experimental models to ex vivo studies. Arthritis Res Ther 2011;13:208.

21 Wei F, Zhou J, Wei X, et al. Activation of Indian hedgehog promotes chondrocyte hypertrophy and upregulation of MMP-13 in human osteoarthritic cartilage. Osteoarthr Cartil 2012;20:755-63.

22 Zhou J, Chen Q, Lanske B, et al. Disrupting the Indian hedgehog signaling pathway in vivo attenuates surgically induced osteoarthritis progression in Col2a1-CreERT2; Ihhfl/fl mice. Arthritis Res Ther 2014;16:R11.

23 Zhou J, Wei X, Wei L. Indian Hedgehog, a critical modulator in osteoarthritis, could be a potential therapeutic target for attenuating cartilage degeneration disease. Connect Tissue Res 2014:55:257-61.

24 Gurdon JB, Bourillot PY. Morphogen gradient interpretation. Nature 2001:413:797-803. 
25 Potter JD. Morphogens, morphostats, microarchitecture and malignancy. Nat Rev Cancer 2007:7:464-74.

26 Park JS, Kim SH, Kim K, et al. Inhibition of notch signalling ameliorates experimental inflammatory arthritis. Ann Rheum Dis 2015;74:267-74.

27 Glasson SS, Askew R, Sheppard B, et al. Deletion of active ADAMTS5 prevents cartilage degradation in a murine model of osteoarthritis. Nature 2005;434: 644-8.

28 Glasson SS, Blanchet TJ, Morris EA. The surgical destabilization of the medial meniscus (DMM) model of osteoarthritis in the 129/SvEv mouse. Osteoarthr Cartil 2007:15:1061-9.

29 Mankin HJ, Dorfman H, Lippiello L, et al. Biochemical and metabolic abnormalities in articular cartilage from osteo-arthritic human hips. J Bone Joint Surg Am 1971;53:523-37.

30 Kamekura S, Hoshi K, Shimoaka T, et al. Osteoarthritis development in novel experimental mouse models induced by knee joint instability. Osteoarthr Cartil 2005;13:632-41.

31 Glasson SS, Chambers MG, Van Den Berg WB, et al. The OARSI histopathology initiative-recommendations for histological assessments of osteoarthritis in the mouse. Osteoarthr Cartil 2010;18(Suppl 3):S17-23.

32 Lin NY, Beyer C, Giessl A, et al. Autophagy regulates TNFalpha-mediated joint destruction in experimental arthritis. Ann Rheum Dis 2013;72:761-8.

33 van der Kraan PM, van den Berg WB. Osteophytes: relevance and biology. Osteoarthr Cartil 2007;15:237-44.

34 Girkontaite I, Frischholz S, Lammi P, et al. Immunolocalization of type X collagen in normal fetal and adult osteoarthritic cartilage with monoclonal antibodies. Matrix Biol 1996:15:231-8.

$35 \mathrm{Yu} \mathrm{X}$, Alder JK, Chun JH, et al. HES1 inhibits cycling of hematopoietic progenitor cells via DNA binding. Stem Cells 2006:24:876-88.

36 Lin NY, Chen CW, Kagwiria R, et al. Inactivation of autophagy ameliorates glucocorticoid-induced and ovariectomy-induced bone loss. Ann Rheum Dis 2016;75:1203-10
37 Sassi N, Laadhar L, Mahjoub M, et al. Expression of Notch family members in cultured murine articular chondrocytes. Biotech Histochem 2009;84:313-20.

38 Mirando AJ, Liu Z, Moore T, et al. RBP-Jk-dependent Notch signaling is required for murine articular cartilage and joint maintenance. Arthritis Rheum 2013;65:2623-33.

39 Kulic I, Robertson G, Chang L, et al. Loss of the Notch effector RBPJ promotes tumorigenesis. J Exp Med 2015;212:37-52.

40 Liu Z, Chen J, Mirando AJ, et al. A dual role for NOTCH signaling in joint cartilage maintenance and osteoarthritis. Sci Signal 2015;8:ra71.

41 Canalis E. Notch signaling in osteoblasts. Sci Signal 2008;1:pe17.

42 Zanotti S, Smerdel-Ramoya A, Canalis E. HES1 (hairy and enhancer of split 1) is a determinant of bone mass. J Biol Chem 2011;286:2648-57.

43 Zhang $\mathrm{H}$, Hilton MJ, Anolik JH, et al. NOTCH inhibits osteoblast formation in inflammatory arthritis via noncanonical NF-kB. J Clin Invest 2014;124:3200-14.

44 Zhen G, Wen C, Jia X, et al. Inhibition of TGF-b signaling in mesenchymal stem cells of subchondral bone attenuates osteoarthritis. Nat Med 2013:19: 704-12.

45 Bai S, Kopan R, Zou W, et al. NOTCH1 regulates osteoclastogenesis directly in osteoclast precursors and indirectly via osteoblast lineage cells. J Biol Chem 2008;283:6509-18.

46 Yamada T, Yamazaki H, Yamane T, et al. Regulation of osteoclast development by Notch signaling directed to osteoclast precursors and through stromal cells. Blood 2003;101:2227-34.

47 Bertrand FE, Angus CW, Partis WJ, et al. Developmental pathways in colon cancer: crosstalk between WNT, BMP, Hedgehog and Notch. Cell Cycle 2012;11:4344-51.

48 Perrimon N, Pitsouli C, Shilo BZ. Signaling mechanisms controlling cell fate and embryonic patterning. Cold Spring Harb Perspect Biol 2012;4:a005975.

49 Radtke F, Raj K. The role of Notch in tumorigenesis: oncogene or tumour suppressor? Nat Rev Cancer 2003;3:756-67.

50 Schreck KC, Taylor P, Marchionni L, et al. The Notch target Hes1 directly modulates Gli1 expression and Hedgehog signaling: a potential mechanism of therapeutic resistance. Clin Cancer Res 2010;16:6060-70. 